\title{
The Effects of Learning Styles on Learning Performance of Blockly Programming with Webduino Online Editor
}

\author{
Peng-Chun Lin, Hsin-Ke Lu* \\ Chinese Culture University, No. 231, Sec. 2, Jianguo S. Rd., Da-an District, Taipei, Taiwan. \\ * Corresponding author. Tel.: +886-2-27005858\#8400; email: sklu@sce.pccu.edu.tw \\ Manuscript submitted March 15, 2019; accepted May 10, 2019. \\ doi: 10.17706/jcp.14.5.373-380
}

\begin{abstract}
Computational Thinking is a significant educational theme in recent years; using programming to develop students' computational thinking is one of crucial training methods so plenty of colleges and universities have set up related curriculum recently to develop their abilities. In light of technological innovations, IoT is nowadays the major concept and technique for promoting intelligent life and the essential knowledge for college and university students. Consequently, the researcher used the online editorWebduino Blockly, in which users could use graphical programming methods to control IoT components allowing them to learn IoT knowledge. Moreover, the researcher utilized UTAUT to investigate their technology acceptance of using this tool and used learning style instrument to evaluate their learning performances of different learning styles. The results showed that performance expectancy had significantly affected behavioral intention; behavioral intention and facilitating conditions had significantly affected use behavior. For learning performance, kinesthetic users' score was significantly higher than auditory users' score; this indicated that using this tool and instructional method could significantly enhance kinesthetic users' learning performance. This study was merely a pilot study; in the future, the researcher expects to investigate learners of all age groups using Blockly programming tool in order to provide more references for instructors.
\end{abstract}

Key words: Computational thinking, leaning style, VARK, UTAUT, blocky programming, Webduino.

\section{Introduction}

D With the rapid technological growth, information technology has become a crucial subject vital to human development and all levels of school have set up various curriculum to develop student's information abilities. During the instructional process, instructors will develop students' computational thinking including logical thinking and systematic thinking by teaching knowledge related to computer science and strengthen their capabilities of application, problem solving, teamwork and innovation of computational thinking by various practices of information technology; among them, the training of programming is an educational method to enhance computational thinking. The major purpose of programming process is to allow learners to compose programs that can solve problems through coding of programming language; i.e., programming will make computational thinking more concrete [1].

In addition to developing thinking abilities, learning new technological applications is another major educational goal of colleges and universities. Furthermore, Knowledge and applications of IoT are highly related to people's daily lives, and therefore the researcher used the online editor Webduino Blockly to teach 
programming and component applications of IoT to develop students' technological knowledge and abilities.

The online editor Webduino Blockly was an IoT program developing platform based on blockly games; it was rare in IoT learning fields and therefore the researcher used UATUT to investigate users' technological acceptance level of this platform for instructors' references. On the other hand, the percentage of actual practice was higher than those of other teaching methods in using actual operations of components and programming to teach IoT knowledge; hence, the researcher also utilized the instrument of VARK learning style to test in order to understand whether this teaching method would affect students' learning performances of different learning styles or not.

\section{Literature Review}

\subsection{Webduino Technique}

Webduino was published by KING KIT TECHNOLOGY CO., LTD. in 2015 and its major concept was integrating Web with Arduino applications to control IoT components by using Web technologies. Due to the characteristics of cross platform and cross device of web technologies, users can utilize them to control various EVB (Evaluation Board) such as Arduino, Raspberry Pi, esp8266...etc., and currently many web designers chose to utilize it to develop IoT applications [2].

It is still a threshold to do webpage programming for people not in IT profession, in order to strengthen computational thinking by simple ways and learn IoT concepts by operating IoT components Webduino team had developed the online editor Webduino Blockly to assist general users such as students and non-IT users to learn. Webduino Blockly is a graphic editor based on Google Blockly; uses can use simple combinations by dragging blocks to control the programs of IoT components and those can be simultaneously transformed to JavaScript so that the operation of actual codes could be understood. The operation example was shown in Fig. 1. The researcher used the online editor Webduino Blockly to teach students taking IoT Basics course and this allowed them to practice programming and operate realistic components for strengthening IoT concepts.

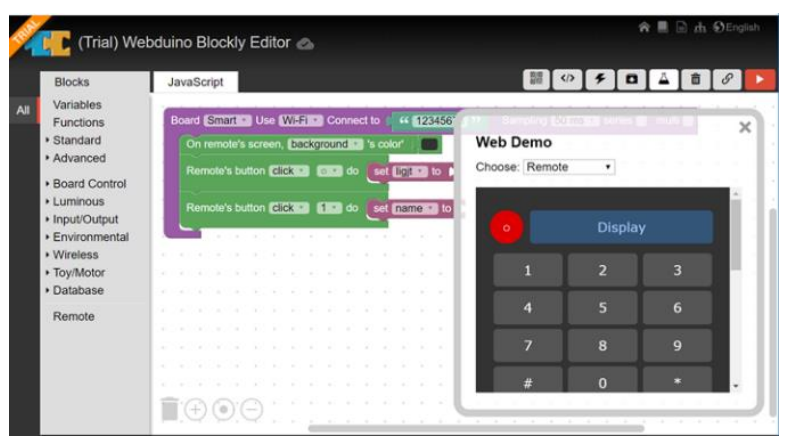

Fig. 1. Example of the Webduino Blockly online editor.

\subsection{Behavioral Intention Models}

Behavioral intention theories are often used as the interpretation models to analyze the adoption of technologies. These theoretical models help us to further understand and reason the intentions and usage behaviors of technologies. Its main structure took attitudes and intentions as dominant factors, which in turn leads to system usage behavior and affects system effectiveness.

In the research fields of technology acceptance, many theories were originated from information system, sociology and psychology. Lu, Lin, Lo and Wu (2012) had analyzed the existing information systems and technology evaluation theories and generalized five theory groups from those similar core concepts or methodologies including Customer Satisfaction Theory, Behavioral Intention Theory, Structuration Theory, 
Innovation Diffusion Theory and Fit Theory [3].

Nevertheless, many researchers regarded that it was insufficient to use only one type of theory for explanation; therefore, Venkatesh, Morris, Davis (2003) did a complete investigation on technology acceptance models and brought up "Unified Theory of Acceptance and Use of Technology" (UTAUT) and also proved that UTAUT had achieved 70\% of explanation [4]. It was completed by integrating the past eight theories related to technology acceptance including Theory of Reasoned Action (TRA), Theory of Planned Behavior (TBP), Technology Acceptance Model (TAM), Social Cognitive Theory (SCT), Model of PC Utilization (MPCU), Motivational Model (MM), Innovation Diffusion Theory (IDT) and Combined TAM and TPB (C-TAMTPB).

\subsection{Learning Styles as Individual Difference}

Each individual had different growing and learning histories, and therefore he/she had his/her own ways to know and understand things and handle excitement or information; these are the so-called individual differences.

Some studies used demographic features as factors for individual differences, while others used cognitive types as differentiator. Lu (2012) integrated learning styles into the study of behavioral intentions of using learning systems. The often applied learning style theories in the field of education are Kolb's model, MyersBriggs, Felder-Silverman's Model and VARK model [3].

The Myers-Briggs Type Indicator (MBTI) classifies learners into four types: extroverts-introverts, sensingintuition, thinking-feeling and judging-perceiving (Harrington \& Loffredo, 2010). Kolb's Learning Styles Inventory model classifies four different styles by two dimensions of thinking preferences (Kolb \& Kolb, 2012). Felder and Silverman's learning styles model has emerged as a wide-used model to be classified in four dimensions: active-reflective (ACT-REF), sensing-intuitive (SEN-SEN), visual-verbal (VIS-VRB) and sequential-global (SEQ-GLO) (Felder \& Silverman, 1988). VARK instrument issued by Fleming in 2001 was a crucial one used by many educators to evaluate learners' learning style; the learning style was categorized into visual, auditory, reading/writing and kinesthetic/tactile styles [5]. In this study, the researcher used the categories and VARK learning style instrument to investigate the effects of learning style on the learning performance of using the online editor Webduino Blockly to learn IoT concepts.

\section{Research Method}

\subsection{Research Framework}

Many research explore the intentions of adopting a learning system, which in turn affects the use behavior and learning outcomes [6]-[8]. Lin, Lu and Liu (2012) propose how to apply the learning type as an e-learning system to replace the use of gender, age and experience to make individual differences. (see Fig. 2) [3].

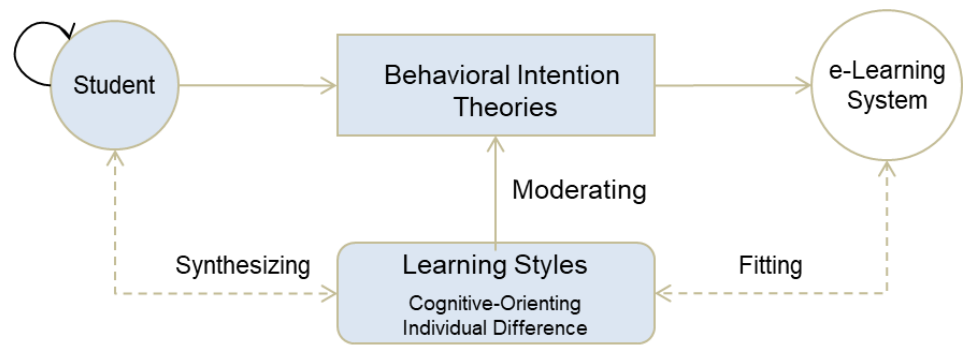

Fig. 2. Learning styles as individual difference factors.

UTAUT model contained four main dimensions and four moderators. The dimensions included Performance Expectancy (PE), Effort Expectancy (EE), Social Influence (SI) and Facilitating Conditions (FC). 
These four dimensions were affected by four moderators including "gender", "age", "experience" and "voluntariness of use". Because UTAUT is based on the two demographic variables of the gender and age mediators, it affects the intention of using the system and its learning effect. However, many studies have found that a number of demographic variables actually influenced behavioral intentions by individual intermediaries, which existed interaction effects among factors. Therefore, many scholars have gradually advocated the use of cognitive-oriented individual differences as an intermediary [7], [9], [10]. The Fig. 3 is one of the modified UTAUT proposed by Lin et al. and researchers used this model to examine the individual differences of users [11].

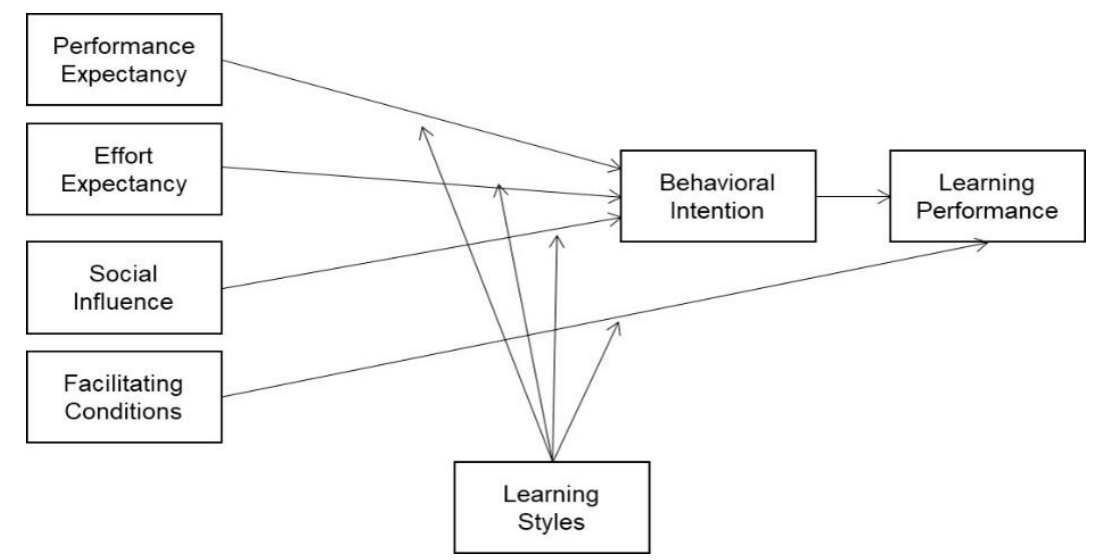

Fig. 3. Modified UTAUT with learning styles.

In this study, PE dimension was defined as "the extent of believing that using this tool can enhance the understanding of IoT concepts"; EE dimension was defined as "the degree of easiness in using this tool"; SI dimension was defined as "the extent of one perceiving that significant others think that he/she should use this tool"; FC dimension was defined as "the extent that one believes that he/she can find out instructions to support using this tool".

The researcher utilized the above four dimensions to investigate leaners' technology acceptance of the online editor Webduino Blockly. However, the participants of this study were all senior students taking IoT Basics course and hence gender percentage was not even; in addition, age and the experiences in using IoT were highly close, and also using this tool was required in the course, and consequently moderators were not investigated in this study.

\subsection{Experimental Design}

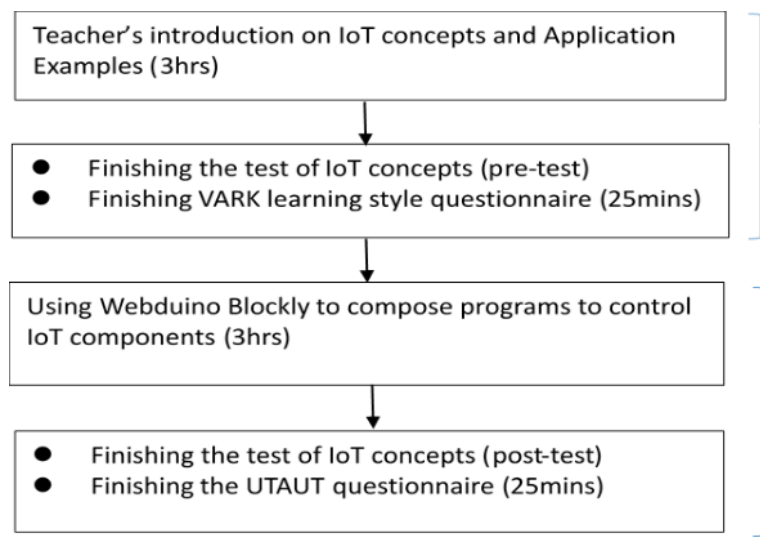

Week 1

Fig. 4. The experimental design of this study. 
The major purpose of this study was to investigate learners' technology acceptance of the online editor Webduino Blockly and the learning performances in using this tool to learn IoT concepts of learners of different learning styles. Therefore, the experimental design was shown in Fig. 4.

\subsection{Research Instruments}

\section{- IoT Concepts Exam}

One research question in this study was to investigate learning performance of users of different learning styles in using the online editor Webduino Blockly to learn IoT concepts. Hence, for evaluating performances before and after the learning activity, one teacher with more than 3 years of instruction designed a "Test of IoT Concepts" and the other professional IoT teacher would reviewed and adjusted it. There were totally 10 items including 5 open questions, 4 multiple-choice questions and 1 multiple-choice question (more than one answer); the highest score was 100 , and the test was scored by the instructor.

\section{- UTAUT Questionnaire}

The researcher used UTAUT model to investigate learners' technology acceptance and hence 24 items in the questionnaire issued by Venkatesh, Morris and Davis (2003) were used including 5 items of Performance Expectancy (PE), 5 items of Effort Expectancy (EE), 4 items of Social Influence (SI), 4 items of Facilitating Conditions (FC), 3 items of Behavioral Intention (BI) and 3 items of Use Behavior (UB) [4] in order to collect their feedbacks.

\section{- Learning Style Questionnaire}

VARK learning style questionnaire was used to evaluate learners' learning style [5]. It contained 16 multiple-choice items (more than one answer) and each included choices of four learning styles of VARK, and they could select $0 \sim 4$ choices meaning that if there was not suitable choice they didn't have to answer and if there were more than 2 choices suitable they could select these two.

\section{- Smart PLS}

Partial Lease Squares (PLS) was used in this study; it was an analyzing technology to investigate or construct a predictive model, particularly better than linear structural relations (LISREL) for analyzing a casual model among latent variables. The merits of PLS included that: (1) it could process multiple dependent and independent variables, (2) it could overcome the problem of multivariate collinearity, (3) it could process interference data and missing values robustly, (4) it could predict precisely on latent variables when response variables were input, (5) it could process reflective indicators and formative indicators simultaneously, (6) it was suitable for a small sample size, (7) it didn't have the limitation of data distribution (Pirouz, 2006). The sample size of this study was small, and therefore the researcher used the path analysis tool smart PLS developed by Ringle, Wende \& Will (2005) to test PLS and hence it wouldn't have the limitations of variable distribution type and sample size; moreover, it would have better predictive and explanatory abilities [12].

\section{- SPSS}

Those learners' learning performances needed to be analyzed in this study, hence the researcher used the statistics software SPSS to conduct the one-way analysis of variance (ANOVA) and also utilized Least Significant Difference (LSD) to do post hoc tests for understanding the differences of the post-test among those learning styles.

\section{Results and Discussions}

\subsection{Participants}

The participants of this study were 53 seniors taking the course of "IoT Basics" from different departments non-related to IoT knowledge and programming. Among them, 50 students were male (94.3\%), 3 students were female (5.7\%), and their ages were between 21 and 24 . 


\subsection{Results of Path Analysis}

In this study, Smart PLS was used to test the UTAUT model, and the proposed model and the results of path analysis were shown in Fig. 5.

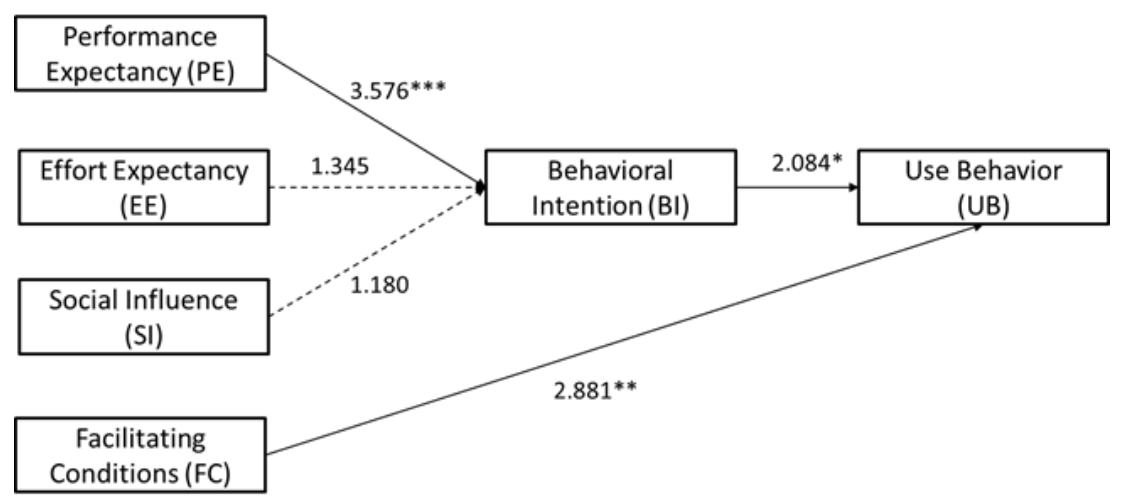

Fig. 5. Results of path analysis in this study.

From Fig. 5, it was known that Performance Expectancy (PE) had significantly affected Behavioral Intention (BI); Facilitating Conditions (FC) and Behavioral Intention (BI) had significantly affected Use Behavior (UB). The possible reason might be that although they had the lectures on IoT basic concepts, they didn't actually compose programs for controlling components, hence they perceived that using this tool did help them to continue learning IoT knowledge due to practices.

Those participants used Webduino Blockly this online editor for the first time, and therefore the results showed that the sufficiency of auxiliary instructions would significantly affect Behavioral Intention (BI), and this agreed with the results of other studies. The significant effect of Behavioral Intention (BI) on Use Behavior (UB) meant that when users thought that this tool was helpful for learning IoT concepts, then they would use it to continue learning advanced knowledge.

Effort Expectancy (EE) and Social Influence (SI) didn't significantly affect Behavioral Intention (BI); the possible reason might be that the experiment period was short so users didn't use it for a long time hence Effort Expectancy (EE) didn't significantly affect Behavioral Intention (BI). For Social Influence (SI), it might be that users used it for the first time so they didn't understand others' opinions about it; hence, it didn't have a significant effect.

\subsection{Analysis of Variance (ANOVA)}

In this study, users' VARK learning styles were investigated, and the results showed that 8 users were visual style (15.09\%), 7 users were auditory style (13.20\%), 16 users were reading/writing style (30.19\%), and 22 users were kinesthetic/tactile style (41.52\%) among 53 users. Furthermore, the researcher had analyzed whether there were significant differences among learning performances of different learning styles or not. In this study, SPSS was used to conduct the homogeneity test of regression slope to make sure whether there were significant differences among pre-test scores of each group or not. For Levene's test result, $p=.201>0.05$; this meant that error variance from the dependent variables of each group was not significantly different; they were homogenous, and analysis of covariance could be conducted further. The results of ANCOVA showed that $F(3,49)=3.11, p=.035<.05$, meaning that post-test scores of each group were different. The researcher then utilized Least Significant Difference (LSD) to conduct a post-hoc analysis and the results indicated that the average post-test score of the kinesthetic group was 6.32, significantly higher than 4.56 of the auditory group. It was possible that the purpose of this tool was using component practice and programming to learn IoT concepts hence the learning willingness and performance of users of kinesthetic 
style would be better; however, lecturing methods might be more suitable for auditory users to understand concepts and this might cause differences of learning performance.

\section{4. $t$-test}

In this study, researchers used the t-test to discuss learning performance of students and the results showed in the Table 1.

Table 1. The Analysis of Pre-test and Post-test Scores in this Study

\begin{tabular}{lllllll}
\hline & Test & $\begin{array}{c}\text { Number of } \\
\text { students }\end{array}$ & $\begin{array}{l}\text { Mean } \\
\text { score }\end{array}$ & $\begin{array}{c}\text { Standard } \\
\text { deviation }\end{array}$ & $\mathrm{t}$ & $\mathrm{p}$ \\
\hline $\begin{array}{l}\text { IoT } \\
\text { concepts } \\
\text { exam }\end{array}$ & Pre-test & 53 & 4.09 & 1.974 & & $\mathrm{p}=.000$ \\
& Post-test & 53 & 5.72 & 1.511 & & $(<0.01)$ \\
\hline \hline
\end{tabular}

The total score of the IoT concepts exam is 10 . The result of t-test analysis indicated that the mean posttest scores were 5.72 which is significantly higher than the pre-test $(p=0.000<.001)$.

\section{Conclusions and Discussions}

The purpose of this study was to investigate learners' technology acceptance of the online editor Webduino Blockly and the learning performance differences in using this tool to learn IoT concepts of learners of different learning styles. In this study, the online editor Webduino Blockly was used as a teaching tool to instruct IoT concepts. The results showed that users felt positively about using this tool to enhance IoT knowledge and it had significantly affected behavioral intention; moreover, its auxiliary instructions and behavioral intention had also significantly affected the follow-up use behaviors.

For learning styles, kinesthetic users had the highest score among all groups in the post test; besides, it was significantly higher than auditory users' score. The results indicated that using this tool to teach learners to learn IoT concepts could enhance kinesthetic users' learning performance. The learning performance results indicated that there were significant differences in the pre- and post-test scores of the students in this study and the average post-test score is higher than the pre-test score. This means the blocky programming online editor can assist students in improving their IoT concepts abilities.

This study was a pilot study whose participants only included college students taking IoT Basics course and the sample size was rather small. In the future, the researcher expects to investigate learners of all age groups using Blockly programming tool in order to provide more references for instructors.

\section{References}

[1] Wing, J. M. (2006). Computational thinking. Communications of the ACM - Self Managed Systems, 49(3), 33-35.

[2] Webduino T. Retrieved November 10, 2018, from https://tutorials.webduino.io/zhtw/docs/basic/index.html

[3] Lu, H. K., Lin, P. C., Lo, C. H., \& Wu, M. Y. (2012). A review of information system evaluation methods. Proceedings of International Proceeding of Computer Science and Information Technology (pp. 243-248).

[4] Venkatesh, V., Morris, M., Davis, G., \& Davis, F. (2003). User acceptance of information technology: Toward a unified view. MIS Quarterly, 27(3), 425-478.

[5] Fleming, N. D.(2001). Teaching and Learning Styles: VARK Strategies.

[6] Price, L. (2004). Individual differences in learning: Cognitive control, cognitive style, and learning style. Educational Psychology, 24(5), 681-698. 
[7] Diseth, Å. (2011). Self-efficacy, goal orientations and learning strategies as mediators between preceding and subsequent academic achievement. Learning and Individual Differences, 21(2), 191-195.

[8] Schmidt, R. (2012). Attention, awareness, and individual differences in language learning. Perspectives on Individual Characteristics and Foreign Language Education, 6, 27.

[9] Felder, R. M., \& Brent, R. (2005). Understanding student differences. Journal of Engineering Education, 94(1), 57-72.

[10] Lin, P. C., Lu, H. K., \& Liu, S. C. (2013). Toward an education behavioral intention model for e-learning systems: An extension of UTAUT. Journal of Theoretical and Applied Information Technology, 47(3), 11201127.

[11] Balakrishnan, V., \& Gan, C. L. (2016). Students' learning styles and their effects on the use of social media technology for learning. Telematics and Informatics, 33(3), 808-821.

[12] Ringle, C. M., Wende, S., \& Will, A. (2005). SmartPLS-Version 2.0. Germany: University at Hamburg. Retrieved November 10, 2018, from http://www.smartpls.de

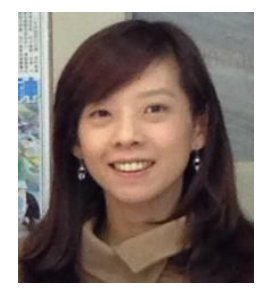

Peng-Chun Lin is an assistant professor of Department of Information Management in Chinese Culture University. She is also the chief officer of the international information certification cooperation center at Chinese Culture University in Taiwan. Her academic interests focus on e-learning, application of simulation learning and recently on optimal network management and corporate structure.

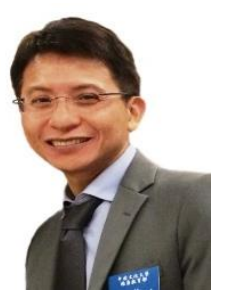

Hsin-Ke Lu is an associate professor and the director of information management (master programs for working learners). He was the chairman of the Association of Continuing Education of Colleges and Universities in Taiwan and the chairman of Cisco Networking Academy. His academic interests focus on e-learning, corporate structure, lifelong learning, and information system planning (enterprise architecture). 